\title{
Validation of the Center for Epidemiological Studies Depression Scale among Korean Adolescents
}

\author{
Eun-Hye $\mathrm{Heo}^{1}$, Kyeong-Sook $\mathrm{Choi}^{2} \bowtie$, Je-Chun $\mathrm{Yu}^{2}$, and $\mathrm{Ji}-\mathrm{Ae} \mathrm{Nam}{ }^{3}$ \\ ${ }^{1}$ Department of Neuropsychiatry, Eulji University Hospital, Daejeon, Republic of Korea \\ ${ }^{2}$ Department of Neuropsychiatry, School of Medicine, Eulji University Hospital, Daejeon, Republic of Korea \\ ${ }^{3}$ Daedeok-gu Mental Health Center, Daejeon, Republic of Korea
}

\begin{abstract}
Objective The Center for Epidemiological Studies Depression Scale (CES-D) is designed to measure the current level of depressive symptomatology in the general population. However, no review has examined whether the scale is reliable and valid among children and adolescents in Korea. The purpose of this study was to test whether the Korean form of the CES-D is valid in adolescents.

Methods Data were obtained from 1,884 adolescents attending grades 1-3 in Korean middle schools. Reliability was evaluated by internal consistency (Cronbach's alpha). Concurrent validity was evaluated by a correlation analysis between the CES-D and other scales. Construct validity was evaluated by exploratory factor and confirmatory factor analyses.

Results The internal consistency coefficient for the entire group was 0.88 . The CES-D was positively correlated with scales that measure negative psychological constructs, such as the State Anxiety Inventory for Children, the Korean Social Anxiety Scale for Children and Adolescents, and the Reynold Suicidal Ideation Questionnaire, but it was negatively correlated with scales that measure positive psychological constructs, such as the Korean version of the Rosenberg Self-Esteem Scale and the Connor-Davidson Resilience Scale-2. The CES-D was examined by three-dimensional exploratory factor analysis, and the three-factor structure of the scale explained $53.165 \%$ of the total variance. The variance explained by factor I was $24.836 \%$, that explained by factor II was $15.988 \%$, and that explained by factor III was $12.341 \%$. The construct validity of the CES-D was tested by confirmatory factor analysis, and we applied the entire group's data using a three-factor hierarchical model. The fit index showed a level similar to those of other countries' adolescent samples.

Conclusion The CES-D has high internal consistency and addresses psychological constructs similar to those addressed by other scales. The CES-D showed a three-factor structure in an exploratory factor analysis. The present findings suggest that the CES-D is a useful and reliable tool for measuring depression in Korean adolescents.

Psychiatry Investig 2018;15(2):124-132
\end{abstract}

Key Words Adolescent psychiatry, Depression, Psychometrics.

\section{INTRODUCTION}

Depressive symptoms are common in adolescents. ${ }^{1}$ The prevalence of adolescent depressive disorders ranges from $1.5 \%$ to $8 \%$ in community and clinical samples, and the lifetime prevalence is estimated to be as high as $20 \% .{ }^{1}$ Depression in adolescents is associated with serious negative outcomes, such as increased risk for substance abuse, school dropout, and suicide attempts. ${ }^{2}$

\footnotetext{
Received: April 30, 2017 Revised: June 16, 2017

Accepted: July 19, 2017 Available online: December 1, 2017

$\triangle$ Correspondence: Kyeong-Sook Choi, MD, PhD

Department of Neuropsychiatry, School of Medicine, Eulji University Hospital, 95 Dunsanseo-ro, Seo-gu, Daejeon 35233, Republic of Korea Tel: +82-42-611-3443, Fax: +82-42-611-3445, E-mail: cksinj@eulji.ac.kr

(c) This is an Open Access article distributed under the terms of the Creative Commons Attribution Non-Commercial License (http://creativecommons.org/licenses/by$\mathrm{nc} / 4.0$ ) which permits unrestricted non-commercial use, distribution, and reproduction in any medium, provided the original work is properly cited.
}

The most commonly used self-report measure of child depression is the Children's Depression Inventory (CDI), which was developed by Kovacs as a downward revision of the Beck Depression Inventory (BDI-I \& II). ${ }^{3}$ However, Hensel and Matson $^{4}$ assessed depression in children aged 6-18 years and demonstrated that age is a significant factor in depression scores, whereas race and gender are not. Older children tend to display more depressive symptomatology, suggesting that the CDI has limited utility for detecting depression among adolescents. The CDI was translated into Korean and validated by Cho and Lee, ${ }^{5}$ and the factor structure of the CDI in children and adolescents was determined by Kim et al. ${ }^{6}$ In that study, children showed a three-factor structure and adolescents showed four factors. Therefore, the factor structure of the CDI differs by developmental level. ${ }^{6}$ The BDI-I \& II are useful for measuring depression, but they are of limited applicability for purposes of research and high-risk group selection. Indeed, 
the BDI is used to evaluate the severity of depression and for screening depression in clinical practice and in the general population of adolescents and adults. ${ }^{7}$

The purpose of the Center for Epidemiologic Studies Depression (CES-D) differs from that of previous depression scales used chiefly for diagnosis at clinical intake and/or for the evaluation of the severity of illness over the course of treatment. The CES-D was designed to measure the current level of depressive symptomatology, with emphasis on the affective component, depressed mood. The symptoms addressed by the CES-D include those on which a diagnosis of clinical depression is based; however, they may also accompany, to some degree, other diagnoses (and "normal"). ${ }^{8}$ Therefore, the purpose of the CES-D is slightly different from that of the BDI-I \& II.

Data on the BDI have shown that not all student and community samples with elevated scores meet the diagnostic criteria for major depression. ${ }^{7}$ If the subjects of a community sample study are primarily adolescents, a scale that measures a major depressive episode may not be sensitive for screening purposes.

Although originally developed to identify depressive symptoms among adults, the CES-D has been widely used to examine depressive symptoms in adolescents. ${ }^{1}$ The reliability, validity, and utility of identifying cases of major depressive disorder using the CES-D and other symptom screening scales have been established in adult populations, ${ }^{8}$ but the same clinical thresholds have not been applied to child and adolescent samples. ${ }^{9}$

The CES-D is a short self-report scale designed to measure the current level of depressive symptomatology in the general population. The major components of depressive symptomatology include depressed mood, feelings of guilt and worthlessness, feelings of helplessness and hopelessness, psychomotor retardation, loss of appetite, and sleep disturbance. ${ }^{8}$ It is a short self-report assessment scale that has been developed for screening purposes and is commonly used in scientific studies. ${ }^{10}$ The advantages of the CES-D are its free use (no need to pay a copyright fee), its translation into many languages, its widespread usage, and its suitability for use in cross-cultural studies. ${ }^{11}$ The CES-D has been validated to screen for depression among adolescent Native Hawaiians and members of other ethnic groups. ${ }^{12}$ The CES-D not only measures depression but also predicts major depression and dysthymia.

The CES-D was validated in adolescents from Vietnam in 2016, ${ }^{13}$ in those from Hong Kong in $2015^{14}$ in those from mainland China in $2013^{15}$ and in those from Turkey in 2013. ${ }^{11}$ Additionally, a factor structure analysis was performed in Filipino-Americans ${ }^{16}$ and American Indians (Native Americans). ${ }^{17}$ In Korea, the CDI, ${ }^{5} \mathrm{BDI}-\mathrm{I},{ }^{18}$ and BDI-II ${ }^{19}$ have been validated in adolescents, but the CES-D has not been validated in adolescents.
The purpose of this study was to test whether the Korean form of the CES-D is an effective tool for identifying depression in adolescents by determining its reliability and validity in an adolescent sample.

\section{METHODS}

\section{Participants}

Data were obtained from students attending grades $1-3$ in middle schools in Korea. We explained the purpose of the study, contents, and procedure to the subjects, who provided written consent. We also sent a communication about the study to students' homes. Students who provided consent whose parent or guardian also provided written informed consent completed self-report questionnaires. This study was approved by the Eulji University Hospital Institutional Review Board (EMC2014-07-009-001).

In total, 1,884 students were included in the study $(1,252$ males (66.5\%) and 632 females (33.5\%). The sample was comprised of $145(7.7 \%)$ first-grade, 1,536 (81.5\%) second-grade, and 203 (10.8\%) third-grade students. There were 43 (2.3\%) 13-year-olds, 318 (16.9\%) 14-year-olds, 1,292 (68.6\%) 15-yearolds, and 204 (10.8\%) 16-year-olds. The demographic characteristic of the sample are presented Table 1.

\section{Measures}

\section{Center for Epidemiological Studies Depression Scale (CES-D)}

The CES-D ${ }^{8}$ consists of 20 items describing a wide range of depressive symptoms. Respondents rated their symptoms in the past week on a $0-3$ scale from "rarely or never ( $<1$ day)" to "most or all of the time (5-7 days)." The total summed score was $0-60{ }^{20}$ The Korean version of CES-D was translated by

Table 1. Demographic distributions of the samples $(\mathrm{N}=1884)$

\begin{tabular}{lr}
\hline Demographics & Number $(\%)$ \\
\hline Sex & \\
Male & $1,252(66.5)$ \\
Female & $632(33.5)$ \\
Grade & \\
1st & $145(7.7)$ \\
2nd & $1,536(81.5)$ \\
3rd & $203(10.8)$ \\
Age & \\
13 & $43(2.3)$ \\
14 & $318(16.9)$ \\
15 & $1,292(68.6)$ \\
16 & $204(10.8)$ \\
\hline
\end{tabular}


Chon and Rhee. ${ }^{21}$ In this study, we used the scales translated by Chon et al. ${ }^{22}$ In that study, the researchers developed a newly integrated version using items contained in three earlier versions currently available in Korea. The Cronbach's alpha among adults was 0.91 , and the scale contained a four-factor structure similar to the original English scale.

\section{State Anxiety Inventory for Children (SAIC)}

The SAIC was developed by Spielberger. ${ }^{23} \mathrm{Cho}$ and $\mathrm{Choi}^{24}$ translated and standardized this scale using Korean elementary school students. The SAIC and Trait Anxiety Inventory for Children each consist of 20 items rated on a scale from 1 to 3 , with total scores ranging from 0 to 60 . Only the SAIC was used in the present study, and Cronbach's alpha was 0.87 .

\section{Korean Social Anxiety Scale for Children and Adolescents (K-SAS-CA)}

Based on translations of the Social Anxiety Scale for Children Revised ${ }^{25}$ and the Social Phobia and Anxiety Inventory for Children, ${ }^{26}$ the K-SAS-CA was constructed and the psychometric properties evaluated by Moon and Oh. ${ }^{27}$ This scale consists of 40 items rated on a 5-point Likert scale. The results of a factor analysis performed by Moon and $\mathrm{Oh}$ indicated that the scale consists of five factors: Performance anxiety, Fear of negative evaluation, Avoidance, Fear of new situations or unfamiliar peers, and Nonassertiveness. Internal consistencies for the subscales have ranged from 0.79 to 0.92 , and construct validity is good.

\section{Reynolds Suicide Ideation Questionnaire (RSIQ)}

We used the self-report RSIQ scale ${ }^{28}$ to assess suicidal ideation among adolescents during the previous month. Shin ${ }^{29}$ validated this scale, which consists of 30 items rated on a scale from 0 to 6, for Koreans. A total score of 180 points is possible, and a higher score indicates more suicidal ideation. In this study, Cronbach's alpha was 0.97

\section{Korean version of the Rosenberg Self-Esteem Scale (K-RSES)}

This scale, which measures self-esteem, consists of 5 positive self-esteem items and 5 negative self-esteem items. This is a self-report measure that relies on a 4-point Likert scale, with higher total scores reflecting higher self-esteem. We used the version translated and validated by Bae et al. ${ }^{30}$ In that study, Cronbach's alpha was 0.90 , and Cronbach's alpha was 0.86 in the present study.

\section{Connor-Davidson Resilience Scale-2 (CD-RISC2)}

The original CD-RISC was developed by Connor and Davi$\operatorname{sion}^{31}$ to assess resilience. This is a self-report scale consisting of 25 items rated on a 5-point Likert scale $(0-4)$, with higher scores reflecting greater resilience. We used the CD-RISC2, which is a shortened version of the CD-RISC that has the advantage of requiring considerably less time to administer while maintaining similar efficiency. The CD-RISC2 includes 2 items that assess whether respondents are "able to adapt to change" and "tend to bounce back after illness or hardship."32 We used the Korean version of the CD-RISC2 validated by Jeong et al. ${ }^{33}$ In their study, Cronbach's alpha was 0.75 , and Cronbach's alpha was 0.73 in the present study.

\section{Statistical analysis}

The reliability of the CES-D was evaluated in terms of internal consistency (Cronbach's alpha). Validity was determined in terms of concurrent validity and construct validity. To investigate concurrent validity, we conducted a correlation analysis to examine whether the CES-D was positively correlated with scales that measure negative psychological constructs and negatively correlated with scales that measure positive psychological constructs. To investigate construct validity, we conducted exploratory factor and confirmatory factor analyses. The confirmatory factor analysis was conducted using a structural equation model to validate the elementary factors produced by the exploratory factor analysis using the Comparative Fit Index (CFI), Tucker-Lewis Index (TLI), Goodness of Fit Index (GFI), and Root Mean Square Error of Approximation (RMSEA). The data were analyzed with SPSS 22 and Amos 22 software (IBM Corp., Armonk, NY, USA).

\section{RESULTS}

\section{Reliability}

\section{Internal consistency}

To examine the reliability of the CES-D, the Cronbach's alpha internal consistency coefficient was computed. In this study, the internal consistency coefficient for the entire group was 0.88 , indicating that the CES-D is reliable for assessing depressive symptoms in the adolescent and general adult population. The results of the item analysis are presented in Table 2, and the correlations between the CES-D items for Korean adolescents are presented in Table 3.

\section{Validity}

\section{Concurrent validity}

The CES-D was positively correlated with scales that measure negative psychological constructs, such as the SAIC, KSAS-CA, and RSIQ, but was negatively correlated with the KRSES and CD-RISC2, which measure positive psychological 
Table 2. Item analysis of the CES-D scale for entire group $(\mathrm{N}=1884)$

\begin{tabular}{|c|c|c|c|c|}
\hline Item & $\mathrm{M}$ & SD & Item-total correlation & Alpha if item deleted \\
\hline 1. Bothered & 0.78 & 0.786 & 0.468 & 0.876 \\
\hline 2. Appetite & 0.42 & 0.658 & 0.391 & 0.878 \\
\hline 3. Blues & 0.29 & 0.599 & 0.580 & 0.873 \\
\hline 4. Good & 1.73 & 0.982 & 0.223 & 0.887 \\
\hline 5. Mind & 0.57 & 0.770 & 0.485 & 0.875 \\
\hline 6. Depressed & 0.38 & 0.707 & 0.678 & 0.869 \\
\hline 7. Effort & 0.51 & 0.762 & 0.623 & 0.870 \\
\hline 8. Hopeful & 1.52 & 0.997 & 0.307 & 0.884 \\
\hline 9. Failure & 0.30 & 0.613 & 0.544 & 0.874 \\
\hline 10. Fearful & 0.28 & 0.593 & 0.624 & 0.872 \\
\hline 11. Sleep & 0.37 & 0.730 & 0.421 & 0.877 \\
\hline 12. Happy & 1.05 & 0.975 & 0.421 & 0.879 \\
\hline 13. Talk & 0.44 & 0.739 & 0.429 & 0.877 \\
\hline 14. Lonely & 0.26 & 0.606 & 0.639 & 0.871 \\
\hline 15. Unfriendly & 0.26 & 0.581 & 0.562 & 0.873 \\
\hline 16. Enjoy & 1.14 & 0.973 & 0.463 & 0.877 \\
\hline 17. Crying & 0.23 & 0.568 & 0.530 & 0.874 \\
\hline 18. Sad & 0.33 & 0.622 & 0.607 & 0.872 \\
\hline 19. Dislike & 0.29 & 0.604 & 0.624 & 0.872 \\
\hline 20. Get going & 0.36 & 0.665 & 0.621 & 0.871 \\
\hline
\end{tabular}

CES-D: Center for Epidemiological Studies Depression Scales, M: mean, SD: standard deviation

constructs (Table 4). This means that the CES-D addresses psychological constructs similar to those addressed by these other measures.

\section{Construct validity}

\section{Exploratory factor analysis}

The construct validity of the CES-D was first tested with an exploratory factor analysis. Principal components analysis with a varimax rotation was applied to all data. The exploratory factor analysis determined that the scale was three dimensional and that the three-factor structure of the scale explained $53.17 \%$ of the total variance. The variance explained by factor I was $24.836 \%$, that by factor II was $15.988 \%$, and that by factor III was $12.341 \%$ (Table 5).

Factor I consisted of ten items: 14 (Lonely), 19 (Dislike), 18 (Sad), 15 (Unfriendly), 17 (Crying), 6 (Depressed), 10 (Fearful), 3 (Blues), 13 (Talk), and 11 (Sleep). Factor II consisted of six items: 1 (Bothered), 5 (Mind), 7 (Effort), 20 (Get Going), 2 (Appetite), and 9 (Failure). Factor III consisted of four items: 16 (Enjoy), 12 (Happy), 8 (Hopeful), and 4 (Good).

\section{Confirmatory factor analysis}

The construct validity of the CES-D was tested by confirmatory factor analysis, and we examined all data using a threefactor hierarchical model. Model A was based on the findings from the present study. The CFI was 0.780, the TLI was 0.725 , the GFI was 0.781 , and the RMSEA was 0.104 . The fit index for the confirmatory factor analysis of the other model, which examined Korean adults, was similar to the present results. The factor loadings for each of the items calculated by the confirmatory factor analysis are presented in Table 6 . The fit schema of the scale to the three-factor structure is presented in Figure 1. Model B was based on the findings of Radloff for an adult sample ${ }^{8}$ and consisted of a four-factor structure: factor I (items 3, 6, 9, 10, 14, 17, and 18), factor II (items 4, 8, 12, and 16), factor III (items 1, 2, 5, 7, 11, 13, and 20), and factor IV (items 15 and 19). We applied this model to Korean adolescents. The results of this model were similar to our results $(\mathrm{CFI}=0.794, \mathrm{TLI}=0.738, \mathrm{GFI}=0.794$, and $\mathrm{RM}$ $\mathrm{SEA}=0.101)$. Model $\mathrm{C}$ by Shin et $\mathrm{al}^{34}$ consisted of four factors: factor I (items 5, 7, 9, 10, 13, 14, 15, 19, and 20), factor II (items 4, 8, 12, and 16), factor III (items 1, 2, 3, 6, and 11), and factor IV (items 17 and 18). The fit indices for this model among Korean adolescents did not differ from those for our 


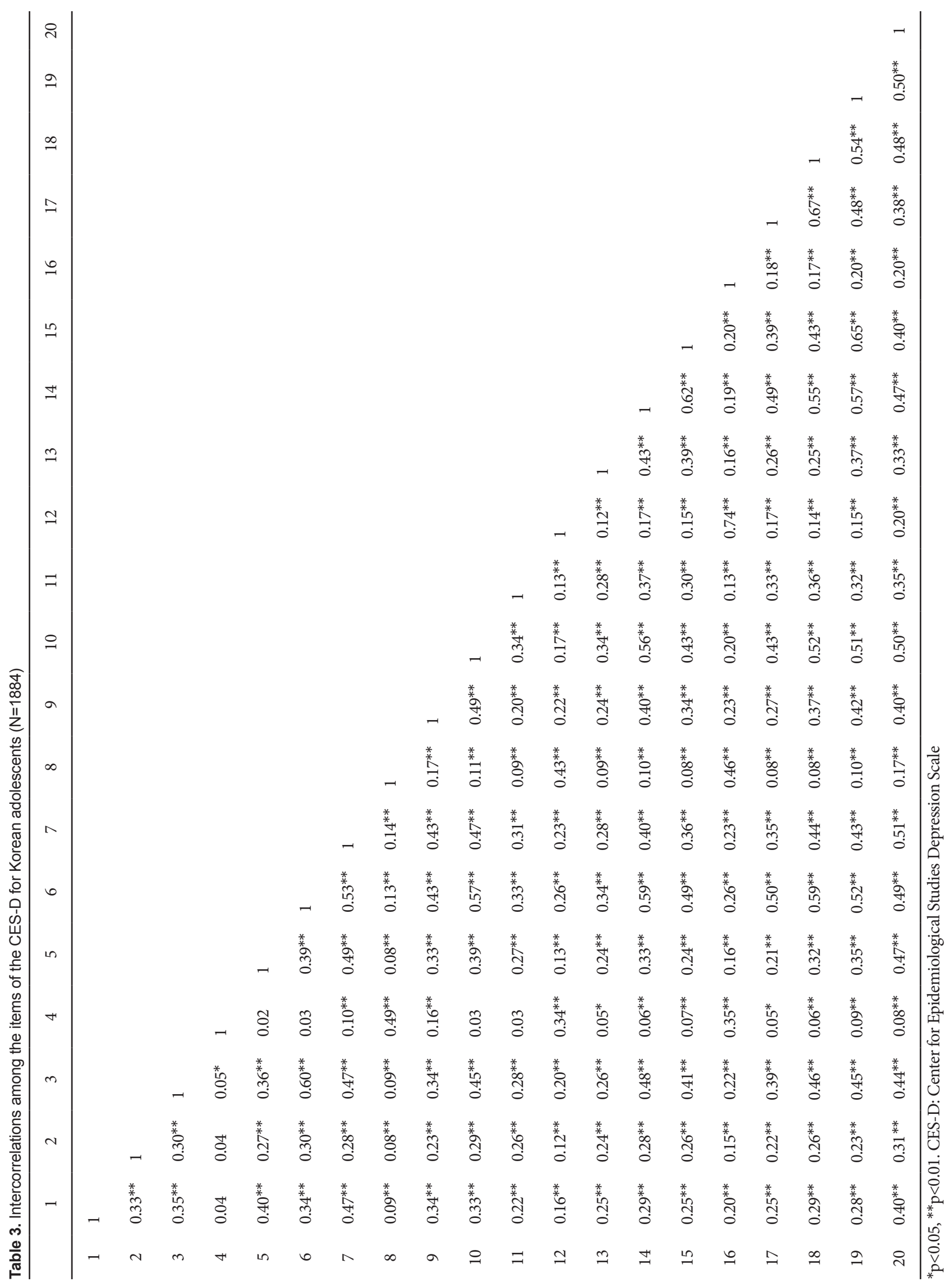


Table 4. Correlation with SAIC, K-SAS-CA, RSIQ, K-RSES, CD-RISC2 ( $\mathrm{N}=1884)$

\begin{tabular}{llllll}
\hline \multicolumn{1}{c}{ Scales } & CES-D & SAIC & K-SAS-CA & RSIQ & K-RSES \\
\hline CES-D & 1 & & & & \\
SAIC & $0.13^{* *}$ & 1 & & & \\
K-SAS-CA & $0.59^{* *}$ & $0.10^{* *}$ & 1 & & \\
RSIQ & $0.60^{* *}$ & $0.10^{* *}$ & $0.47^{* *}$ & 1 & 1 \\
K-RSES & $-0.67^{* *}$ & $-0.25^{* *}$ & $-0.46^{* *}$ & $-0.50^{* *}$ & $0.51^{* *}$ \\
CD-RISC2 & $-0.41^{* *}$ & $-0.14^{* *}$ & $-0.33^{* *}$ & $-0.20^{* *}$ & 1 \\
\hline
\end{tabular}

${ }^{* *} \mathrm{p}<0.01$. CES-D: Center for Epidemiological Studies Depression Scale, SAIC: State Anxiety Inventory for Children, K-SAS-CA: Korean Social Anxiety Scale for Children and Adolescents, RSIQ: Reynolds Suicide Ideation Questionnaire, K-RSES: Korean version of the Rosenberg SelfEsteem Scale, CD-RISC2: Connor-Davidson Resilience Scale-2

Table 5. Factor loading of CES-D items for entire sample $(\mathrm{N}=1884)$

\begin{tabular}{lccc}
\hline \multicolumn{1}{c}{ Item } & Factor I & Factor II & Facotr III \\
\hline 14 (Lonely) & $\mathbf{0 . 7 8 6}$ & 0.213 & 0.058 \\
19 (Dislike) & $\mathbf{0 . 7 5 2}$ & 0.226 & 0.071 \\
18 (Sad) & $\mathbf{0 . 7 4 8}$ & 0.224 & 0.044 \\
15 (Unfriendly) & $\mathbf{0 . 7 4 7}$ & 0.122 & 0.068 \\
17 (Crying) & $\mathbf{0 . 7 3 3}$ & 0.078 & 0.075 \\
6 (Depressed) & $\mathbf{0 . 6 7 8}$ & 0.397 & 0.101 \\
10 (Fearful) & $\mathbf{0 . 6 0 5}$ & 0.424 & 0.058 \\
3 (Blues) & $\mathbf{0 . 5 2 8}$ & 0.430 & 0.078 \\
13 (Talk) & $\mathbf{0 . 4 7 1}$ & 0.216 & 0.072 \\
11 (Sleep) & $\mathbf{0 . 4 3 7}$ & 0.267 & 0.037 \\
1 (Bothered) & 0.123 & $\mathbf{0 . 7 4 0}$ & 0.068 \\
5 (Mind) & 0.186 & $\mathbf{0 . 7 3 6}$ & 0.014 \\
7 (Effort) & 0.362 & $\mathbf{0 . 6 6 9}$ & 0.127 \\
20 (Get Going) & 0.463 & $\mathbf{0 . 5 6 9}$ & 0.079 \\
2 (Appetite) & 0.198 & $\mathbf{0 . 5 0 9}$ & 0.043 \\
9 (Failure) & 0.379 & $\mathbf{0 . 4 6 4}$ & 0.197 \\
16 (Enjoy) & 0.139 & 0.173 & $\mathbf{0 . 8 0 4}$ \\
12 (Happy) & 0.109 & 0.149 & $\mathbf{0 . 7 9 8}$ \\
8 (Hopeful) & 0.037 & 0.047 & $\mathbf{0 . 7 6 5}$ \\
4 (Good) & 0.027 & -0.043 & $\mathbf{0 . 6 9 7}$ \\
\hline Eigen-value & 4.967 & 3.198 & 2.468 \\
Variance explained (\%) & 24.836 & 15.988 & 12.341 \\
\hline
\end{tabular}

CES-D: Center for Epidemiological Studies Depression Scale model (CFI=0.794, TLI=0.738, GFI=0.784, RMSEA=0.101). Model D, which was developed by Kim et al. ${ }^{35}$ was comprised of three factors: factor I (items 1, 2, 3, 5, 6, 7, 8, 9, 10, 11, and 13), factor II (items 4,12 , and 16), and factor III (items 14, $15,17,18,19$, and 20). The fit of this model was similar to that estimated for the Korean adolescents (CFI $=0.737$, TLI $=0.672$, $\mathrm{GFI}=0.738$, RMSEA=0.113). Model E was developed by Chon et al. ${ }^{22}$ and consisted of four factors: factor I (items 1, 2, $3,5,6,7,13$, and 20), factor II (items $4,8,12$, and 16), factor III (items 9, 14, 15, and 19), and factor IV (items 10, 11, 17, and 18). Model E showed similar results (CFI $=0.788$, TLI $=0.731$, $\mathrm{GFI}=0.789$, RMSEA=0.103). Based on these findings, we concluded that these models produced very similar results among Korean adolescents.

\section{DISCUSSION}

In the reliability analysis, the Cronbach's alpha in our study was 0.88 , which was similar to the figures of 0.85 for the general population reported in Radloff's study, ${ }^{8}$ of 0.89 for an adult sample in Korea reported by Chon and Rhee, ${ }^{21}$ and of 0.80 for the Korean adult group studied by Shin et al. ${ }^{34}$ These results indicate that the CES-D shows similar internal consistency among Korean adolescent and adult groups.

In the concurrent validity analysis, the CES-D was positively associated with the SAIC, which measures state anxiety, the K-SAS-CA, which measures social anxiety, and the RSIQ, which measures suicidal ideation. Additionally, the CES-D

Table 6. Fit indices for confirmatory factor analysis $(\mathrm{N}=1884)$

\begin{tabular}{cllccccc}
\hline Model & \multicolumn{1}{c}{ Study } & \multicolumn{1}{c}{$\chi^{2}$} & df & CFI & TLI & GFI & RMSEA \\
\hline Model A & Heo, Choi & $3,577.714$ & 168 & 0.780 & 0.725 & 0.781 & 0.104 \\
Model B & Radloff $^{8}$ & $3,360.825$ & 165 & 0.794 & 0.738 & 0.794 & 0.101 \\
Model C & Shin et al. $^{34}$ & $3,360.825$ & 165 & 0.794 & 0.738 & 0.784 & 0.101 \\
Model D & Kim et al. $^{35}$ & $4,239.103$ & 168 & 0.737 & 0.672 & 0.738 & 0.113 \\
Model E & Chon et al. $^{22}$ & $3,445.369$ & 165 & 0.788 & 0.731 & 0.789 & 0.103 \\
\hline
\end{tabular}

CFI: Comparative Fit Index, TLI: Tucker Lewis Index, GFI: Goodness of Fit Index, RMSEA: Root Mean Square Error of Approximation 


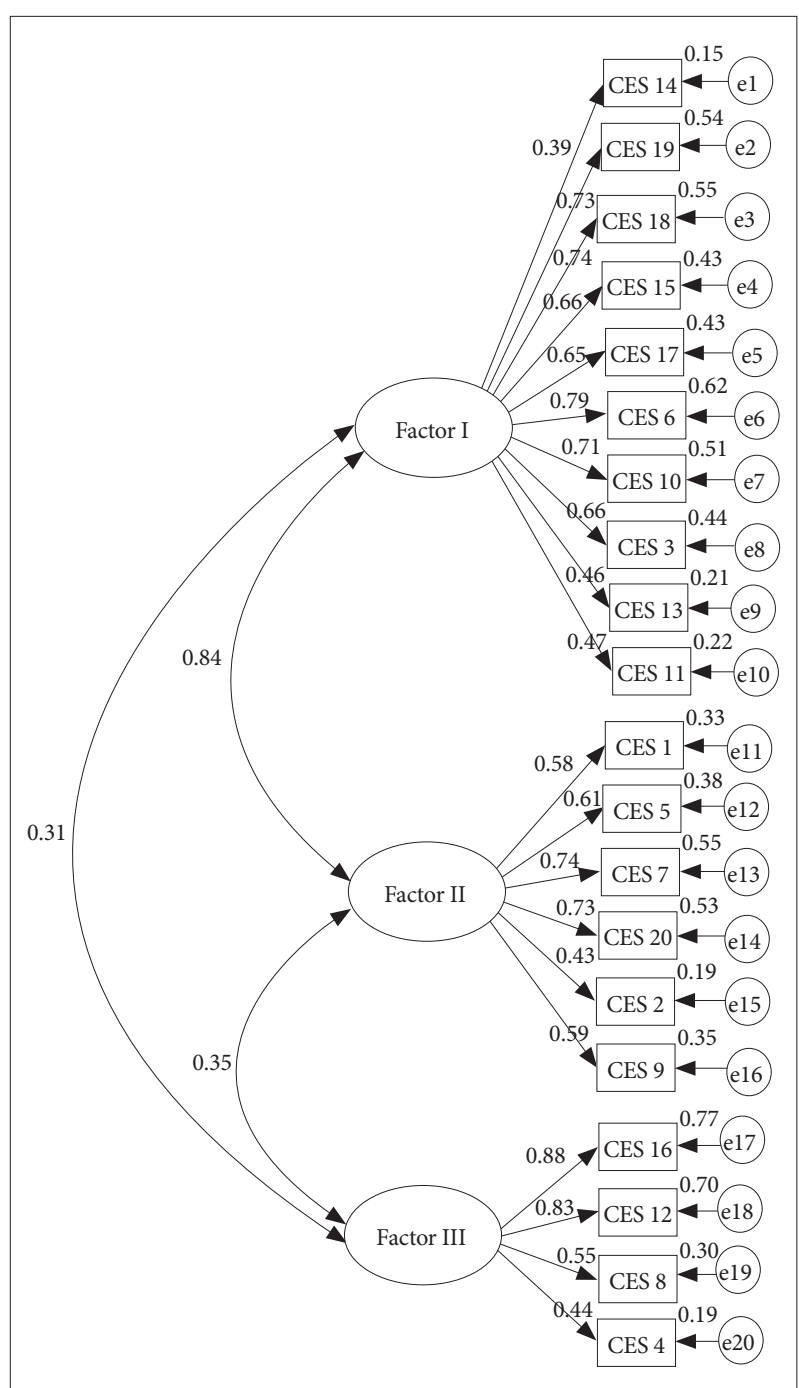

Figure 1. Confirmatory factor analysis of the three factor model for CES-D. CES-D: Center for Epidemiological Studies Depression Scale.

was negatively associated with the K-RSES, which measures self-esteem, and the CD-RISC2.

Principal components factor analysis of the 20 -item scale in this study showed that the CES-D has a three-factor structure. Several studies have examined whether the factor structure of the CES-D differs among adolescents as a function of ethnic group. Radloff (1977) reported similar four-factor solutions among European-Americans and African-Americans, and this has been replicated in other studies. ${ }^{37}$ In Radloff's study, factor IV, Interpersonal (unfriendly, dislike), was a separate and independent factor. In our study, factor IV, Interpersonal, was integrated with factor I, Depressed Affect. In Radloff's study, items 11 (Sleep) and 13 (Talk) were included in Somatic and Retarded activity. However, in our study, these items belonged in factor I, Depressed affect. Items 11 and 13 were in different categories, but, occasionally, both statements fell into different categories.

Item 9 (Failure) was in the Somatic factor in our study, whereas it was in Depressed Affect in Radloff's study. In Shin et al. ${ }^{34}$ and Chon and Rhee ${ }^{21}$ model of Korean adults, the Positive Affect Factor [items 4 (Good), 8 (Hopeful), 12 (Happy), and 16 (Enjoy)] was identical to that in our model; however, other factors did not correspond with those in our model.

Three factors were examined in the factorial structure of the CES-D in American Indian adolescents. ${ }^{17}$ Similar studies have suggested that the four-factor structure reported by Radloff ${ }^{8}$ may not be appropriate for some samples of other ethnicities. Other studies conducted in Asian-American, Hispanic-American, and Native American samples suggested three factors. ${ }^{38-40}$ Three dimensions were also reported in a study of mainland Chinese adolescents. ${ }^{15}$

Two factors provided a reasonably good fit in Filipino-American adolescents; ${ }^{16}$ factor I combined Depressed Affect, Somatic Retardation, and interpersonal items; and factor II consisted of the remaining four positive-affect items. The factor solutions for Korean- and Filipino-Americans in Kuo's study $y^{41}$ also combined Depressed Affect and Somatic Retardation items and consisted of only two factors.

The fit indices from the confirmatory factor analysis in our study were slightly low, but the fit indices were similar to those in another study that examined the validity of the CES-D in adolescents. Among Vietnam adolescents, the CFI was 0.89, the TLI was 0.87 , and the RMSEA was $0.884 .{ }^{13}$ The RMSEA showed a mediocre fit, but the CFI and TLI were $<0.90$. Considering the limitations of that and our study, the goodness of fit from the confirmatory analysis in adolescents differed by ethnicity, especially with regard to differences between respondents from the east and west. The CES-D was first developed with Caucasians, so this scale may not reflect ethnic and cultural differences. As is the case with American Indian adolescents, ${ }^{17}$ the goodness-of-fit index for the three-factor model vs. Radloff's original four-factor structure model was 0.966 . The three-factor structure combined the Somatic and Depressed factors (similar to studies of other ethnic populations) with a single-factor structure. ${ }^{17}$ As these findings suggest that adolescents' responses differ according to ethnicity, ${ }^{16}$ the use of the CES-D in different adolescent ethnic groups requires further study.

Taken together, the validity and reliability studies demonstrate that the Korean form of the CES-D is as effective in adolescents as it is in adults. The scale is available free of charge and is suitable for screening purposes. ${ }^{11}$

This study is the first to determine whether the Korean form of the CES-D is reliable and can be used effectively in adolescents.

Some limitations of this study should be discussed. We did 
not retest depression among adolescents using the CES-D, so we were unable to evaluate the reliability of CES-D over time. We did not compare the CES-D to other depression scales. We did not examine adolescents of all ages, and the sample was drawn from only one location. At the beginning of the data analysis, we confirmed the normal distribution of the data through the Kolmogorov-Smirnov test, but the data did not form a normal distribution. Additionally, we reanalyzed the the data by performing log transformation to adjust the data, but the results were unchanged. The research subject is limited to a certain area and some students and not large in number, so it may not generalized. It is possible that this could not have been significant statistically in confirmatory factor analysis and that the item 2, 4, 8 showed a low correlation in item-total correlation. Therefore, we will have to do more work and identify more evidence through research on larger numbers and different populations.

The CES-D is a useful and reliable measure of depression in Korean adolescents, but the fit indices from the confirmatory factor analysis were slightly low, and the responses differed among ethnicities. ${ }^{16}$

Thus, the CES-D requires further study to determine its concurrent validity with other depression scales, such as the CDI and BDI-II, in adolescents and to examine whether the results of the CES-D are stable across developmental stages. The CESD-R was developed by Dam and Earleywine in 2011. ${ }^{42}$ The CESD-R Korean version was translated and validated in patients and healthy controls by Lee et al. $^{43}$ in 2016 . Further studies measuring depression using the CES-D-R in adolescents will be needed; indeed, comparisons between the original and revised versions of the CES-D will enable researchers to select the most valid and useful tools to measure adolescent depression.

\section{Acknowledgements}

This research was supported by EMBRI Grants 2014EMBRI DI0003 from the Eulji University.

\section{REFERENCES}

1. Rushton JL, Forcier M, Schectman RM. Epidemiology of depressive symptoms in the National Longitudinal Study of Adolescent Health. J Am Acad Child Adolesc Psychiatry 2002;41:199-205.

2. Subramaniam G, Harrell P, Huntley E, Tracy M. Beck depression inventory for depression screening in substance-abusing adolescents. J Subst Abuse Treat 2009;37:25-31.

3. Reynobls WM, Anderson G, Bartell N. Measuring depression in children: a multimethod assessment investigation. J Abnorm Child Psychol 1985;13:513-526.

4. William J, Helsel JL. The assessment of depression in children: the internal structure of the Child Depression Inventory (CDI). Behav Res Ther 1984;22:289-298.

5. Cho SC, Lee YS. Development of the Korean form of the Kovacs' Children's Depression Inventory. J Korean Neuropsychiatr Assoc 1990;29: 943-956.
6. Kim EK, Yang JW, Chung YS, Hong SD, Kim JH. Factor structure of the Children's Depression Inventorty (CDI) in children and adolescents. Korean J Clin Psychol 2005;24:693-707.

7. Whisman MA, Perez JE, Ramel W. Factor structure of the Beck Depression Inventory-Second Edition (BDI-II) in a student sample. J Clin Psychol 2000;56:545-551.

8. Radloff LS. The CES-D Scale: A Self-Report Depression Scale for Research in the General Population. Appl Psychol Meas 1977;1:385-401.

9. Stockings E, Degenhardt L, Lee YY, Mihalopoulos C, Liu A, Hobbs M, et al. Symptom screening scales for detecting major depressive disorder in children and adolescents: a systematic review and meta-analysis of reliability, validity and diagnostic utility. J Affect Disord 2015;174: 447-463.

10. Orme JG, Reis J, Herz EJ. Factorial and discriminant validity of the Center for Epidemiological Studies Depression (CES-D) scale. J Clin Psychol 1986;42:28-33.

11. Tatar A, Kayiran S, Saltukoglu G, Ozkut E, Emeksiz M. Analysis of the Center for Epidemiologic Studies Depression Scale (CES-D) in children and adolescents from the perspective of the item response theory. Klinik Psikofarmakoloji Bulteni-Bull Clin Psychopharmacol 2013;23:242-253.

12. Prescott CA, McArdle JJ, Hishinuma ES, Johnson RC, Miyamoto RH, Andrade NN, et al. Prediction of major depression and dysthymia from CES-D scores among ethnic minority adolescents. J Am Acad Child Adolesc Psychiatry 1998;37:495-503.

13. Nguyen HT, Kitner-Triolo M, Evans MK, Zonderman AB. Factorial invariance of the CES-D in low socioeconomic status African Americans compared with a nationally representative sample. Psychiatry Res 2004;126:177-187.

14. Chin WY, Choi EP, Chan KT, Wong CK. The psychometric properties of the center for epidemiologic studies depression scale in Chinese primary care patients: factor structure, construct validity, reliability, sensitivity and responsiveness. PLoS One 2015;10:e0135131.

15. Wang M, Armour C, Wu Y, Ren F, Zhu X, Yao S. Factor structure of the CES-D and measurement invariance across gender in Mainland Chinese adolescents. J Clin Psychol 2013;69:966-979.

16. Edman JL, Danko GP, Andrade N, McArdle JJ, Foster J, Glipa J. Factor structure of the CES-D (Center for Epidemiologic Studies Depression Scale) among Filipino-American adolescents. Soc Psychiatry Psychiatr Epidemiol 1999;34:211-215.

17. Dick RW, Beals J, Keane EM, Manson SM. Factorial structure of the CES-D among American Indian Adolescents. J Adolesc 1994;17:73-79.

18. Park HJ, Kim HN, Kim IB, Jeon SA. Reliability of the Beck Depression Inventory in adolescence. Korean J Fam Med 2000;21:244-253.

19. Lee EH, Lee SJ, Hwang ST, Hong SH, Kim JH. Reliability and Validity of the Beck Depression Inventory-II among Korean Adolescents. Psychiatry Investig 2017;14:30-36.

20. Verhoeven M, Sawyer MG, Spence SH. The factorial invariance of the CES-D during adolescence: are symptom profiles for depression stable across gender and time? J Adolesc 2013;36:181-190.

21. Chon KK, Rhee MK. Preliminary Development of Korean Version of CES-D. Korean J Clin Psychol 1992;11:65-76.

22. Chon KK, Choi SC, Yang BC. Integrated Adaptation of CES-D in Korea. Korean J Health Psychol 2001;6:59-76.

23. Spielberger CD, Gorsuch RL, Lushene RE. Manual for the State-Trait Anxiety Inventory. CA: Consulting Psychologists Press; 1970.

24. Cho S, Choi J. Development of the Korean form of the state-trait anxiety inventory for children. Seoul J Psychiatry 1989;14:150-157.

25. La Greca AM, Stone WL. Social anxiety scale for children-revised: factor structure and concurrent validity. J Clin Child Psychol 1993;22:17-27.

26. Beidel DC, Turner SM, Morris TL. A new inventory to assess childhood social anxiety and phobia: the social phobia and anxiety inventory for children. Psychol Assess 1995;7:73-79.

27. Moon HS, Oh KJ. A validation study of the Korean Social Anxiety Scale for children and adolescents. Korean J Clin Psychol2002;21:429-443.

28. Reynolds WM. Psychometric characteristics of the Adult Suicidal Ide- 
ation Questionnaire in college students. J Pers Assess 1991;56:289-307.

29. Shin MS. (An) empirical study of the mechanism of suicide: validation of the scale for escape from the self. Seoul: Yonsei University; 1992.

30. Bae HN, Choi SW, Yu JC, Lee JS, Choi KS. Reliability and validity of the Korean version of the Rosenberg Self-Esteem Scale (K-RSES) in adult. J Korean Soc Dep Bip Dis 2014;12:43-49.

31. Connor KM, Davidson JR. Development of a new resilience scale: the Connor-Davidson Resilience Scale (CD-RISC). Depress Anxiety 2003; 18:76-82.

32. Vaishnavi S, Connor K, Davidson JR. An abbreviated version of the Connor-Davidson Resilience Scale (CD-RISC), the CD-RISC2: psychometric properties and applications in psychopharmacological trials. Psychiatry Res 2007;152:293-297.

33. Jeong HS, Kang I, Namgung E, Im JJ, Jeon Y, Son J, et al. Validation of the Korean version of the Connor-Davidson Resilience Scale- 2 in firefighters and rescue workers. Compr Psychiatry 2015;59:123-128.

34. Shin SC, Kim MK, Yun KS, Kim JH, Lee MS, Moon SJ, et al. The Center for Epidemiologic Sutides-Depression Scale (CES-D): its use in Korea. J Korean Neuropsychiatr Assoc 1991;30:752-767.

35. Kim JY, Choi SM, Seo GH, Cho SJ, Kim JK, Cho MJ. Trans-Cultural Difference in Expressing Depressive Symptoms in Koreans. Korean J Psychopathol 2000;9:78-87.
36. Sung KM. Comparison of CES-D and RADS-2-K in Measuring Adolescent Depression. J Korean Soc Sch Health 2016;29:53-62.

37. Clark VA, Aneshensel CS, Frerichs RR, Morgan TM. Analysis of effects of sex and age in response to items on the CES-D scale. Psychiatry Res 1981;5:171-181.

38. Beals J, Manson SM, Keane EM, Dick RW. Factorial structure of the Center for Epidemiologic Studies-Depression Scale among American Indian college students. Psychol Assess J Consult Clin Psychol 1991; 3:623-627.

39. Guarnaccia PJ, Angel R, Worobey JL. The factor structure of the CES-D in the Hispanic Health and Nutrition Examination Survey: the influences of ethnicity, gender and language. Soc Sci Med 1989;29:85-94.

40. Ying YW. Depressive symptomatology among Chinese-Americans as measured by the CES-D. J Clin Psychol 1988;44:739-746.

41. Kuo WH. Prevalence of depression among Asian-Americans. J Nery Ment Dis 1984;172:449-457.

42. Van Dam NT, Earleywine M. Validation of the Center for Epidemiologic Studies Depression Scale-Revised (CESD-R): pragmatic depression assessment in the general population. Psychiatry Res 2011;186:128-132.

43. Lee S, Oh ST, Ryu SY, Jun JY, Lee KS, Lee E, et al. Validation of the Korean version of Center for Epidemiologic Studies Depression ScaleRevised (K-CESD-R). Korean Psychosom Soc 2016;24:83-93. 